\title{
RISULTATI DI UN ANNO DI MISURE ESEGUITE A PAVIA, A CIELO SERENO, CON IL DOSIMETRO PER L'ULTRAVIOLETTO
}

\author{
G. Aliverti - G. Pannocchia
}

Dall'ottobre 1944 al settembre 1945 è stato possibile eseguire a Pavia, presso l'Osservatorio Geofisico, una serie di osservazioni con il dosimetro per l'ultravioletto, osservando ogni ora circa, durante le giornate perfettamente serene verificatesi in quell'epoca; in totale durante 41 giorni. Il dosimetro, di proprietà dell'Istituto di Fisica terrestre e Geodesia dell'Universitì di Torino, è un apparecchio della I. G. Farbenindustrie Aktiengesellschaft a tubo di quarzo, ultimo modello, ed è molto diffuso per scopi climatici e bioclimatici; le sue indicazioni si possono ritenere esatte entro il $10 \%$. Lo scopo che volevamo raggiungere era appunto quello di precisare uno dei caratteri del clima di Pavia che finora non era stato preso in considerazione.

Per ogni misura eseguita è stata calcolata la corrispondente altezza di sole e tutte le misure di un mese sono state rappresentate, in funzione dell'altezza del sole, sopra un unico foglio millimetrato, così che per ciascun mese si è poi potuto tracciare, fra i punti osservati, una linea rappresentante la media intensità della radiazione $U V$ (diretta + diffusa) segnalata dal dosimetro, in funzione dell'altezza del sole. Una unica ascissa è stata attribuita a intensità osservate nella mattina o nel pomeriggio con la medesima altezza di sole; e ciò in considerazione del fatto che il grado di precisione delle misure non è molto elevato come già si è detto. L'insieme dei risultati è sintetizzato dai numeri della tabella I, numeri letti sulle curve medie suddette.

Dalla tabella I si ricavano gli andamenti annui per altezza di sole costante, leggrendo i numeri delle colonne verticali, e gli andamenti mensili per altezza variabile, leggendo i numeri delle righe orizzontali.

Ciascuna curva media mensile è stata planimetrata per calcolare l'energia diurna totale, relativa a ciascun mese, della radiazione $U V$ 
griungente al suolo a Pavia; la fig. 1 rappresenta in unità arbitrarie i risultati del calcolo in questione; essa indica un valore massimo, della energia $U V$ ricevuta, in corrispondenza al mese di maggio. Ma a proposito di questo andamento della curva dell'energia è da notare che al variare dell'altezza del sole si sposta il baricentro dello spettro $U V$ giungente al dosimetro. $\mathrm{E}$ allontanandosi di poco da $i=0,3 \|$ verso valori maggiori di lamda, l'enermia portata dalla radiazione solare diventa anche 100 e più volte maggiore e quindi il massimo dell'ener-

\section{Tabella I}

Intensità della radiazione UV in unità arbitrarie (anno 1944-45, Pavia)

\begin{tabular}{|c|c|c|c|c|c|c|c|c|c|c|c|c|}
\hline $\begin{array}{l}\text { Altezza del } \\
\text { sole }\end{array}$ & $10^{\circ}$ & $15^{\circ}$ & $20^{\circ}$ & $25^{n}$ & $30^{\prime \prime}$ & $3 \overline{5}^{\prime \prime}$ & $40^{\prime \prime}$ & $45^{\circ}$ & $50^{\prime \prime}$ & $55^{\prime \prime}$ & $60^{\circ \prime}$ & $65^{\circ}$ \\
\hline Gennaio & 2,7 & 3,9 & 5,2 & 6,3 & & & & & & & & \\
\hline Febbraio & 1,4 & 2,4 & 3,6 & 5,3 & 7,4 & 8,4 & & & & & & \\
\hline Marzo & 1,0 & 1,8 & 3,0 & 4,6 & 6,6 & 10,1 & 15,8 & 21,4 & & & & \\
\hline Aprile & 1,0 & 1,6 & 2,6 & 4,1 & 6,6 & 9,6 & $1+, 1$ & 20,4 & 28,4 & 32,8 & & \\
\hline Maggio & 0,8 & 1,2 & 2,1 & 4,0 & 6,8 & 9,4 & 13,1 & 17,6 & 22,6 & 28,7 & $3 \cdot 1,4$ & 37,7 \\
\hline Giugno & - & 1,3 & 2,4 & 3,6 & 5,9 & 8,6 & 12,2 & 16,4 & 20,7 & $2 \cdot 1,4$ & 27,0 & 28,6 \\
\hline Luglio & 0,5 & 1,4 & 2,8 & 3,9 & 5,8 & 8,3 & 11,1 & 11,6 & 18,2 & 21,8 & 25,5 & 27,4 \\
\hline Agosto & 0,8 & 1,8 & 2,8 & 3,8 & 6,0 & 9,2 & 13,4 & 17,8 & 22,8 & 27,4 & 29,6 & \\
\hline Settembre & 1,4 & 1,7 & 2,6 & 4,7 & 7,8 & 10,8 & 13,1 & 14,5 & 15,4 & & & \\
\hline Ottobre & 1,4 & 2,1 & 3,7 & 5,4 & 7,6 & 9,3 & 10,4 & & & & & \\
\hline Novembre & 1,8 & 2,2 & 3,3 & 4,3 & & & & & & & & \\
\hline media & 1,3 & 1,9 & 3,1 & 4,6 & 6,6 & 9,3 & 12,9 & 17.5 & 21.3 & $2 \bar{\imath} .0$ & 29,1 & 31,5 \\
\hline
\end{tabular}

gia dedotto dalle misure in questione è di complicala interpretazione; inoltre va tenuto presente anche l'andamento della curva di sensibilità del dosimetro che cade rapidamente verso zero nei pressi di $\lambda=0,32 \mu$.

Dai dati sperimentali non è possibile il calcolo di un fattore di trasmissione non soltanto per le ragioni ora citate ma anche perché il dosimetro non misura soltanto la radiazione diretta ma anche la diffusa e per piccoli valori di $h$ soprattutto il calcolo non ha senso; tuttavia, per le altezze maggiori, per le quali è da ammettere che la 
radiazione diffusa sia piccola in confronto della diretta, questo calcolo indica chiaramente un massimo della radiazione $U V$ di lunghezza d'onda molto corta nei mesi primaverili, soprattutto in maggio poiché soprattutto in quei mesi e non d'estate si ottengono, per altezze del sole sopra i $40^{\circ}$, i valori piì bassi del fattore di trasmissione e precisamente dell'ordine di 0,05 . A proposito di questi valori è da ricordare che per Washington, Coblenz e Stair danno 0,06 per $\lambda=0,300 \mu$ e 0,12 per $\lambda=0,305 \mu$.

Nella figura 2 sono rappresentati graficamente gli andamenti lun-

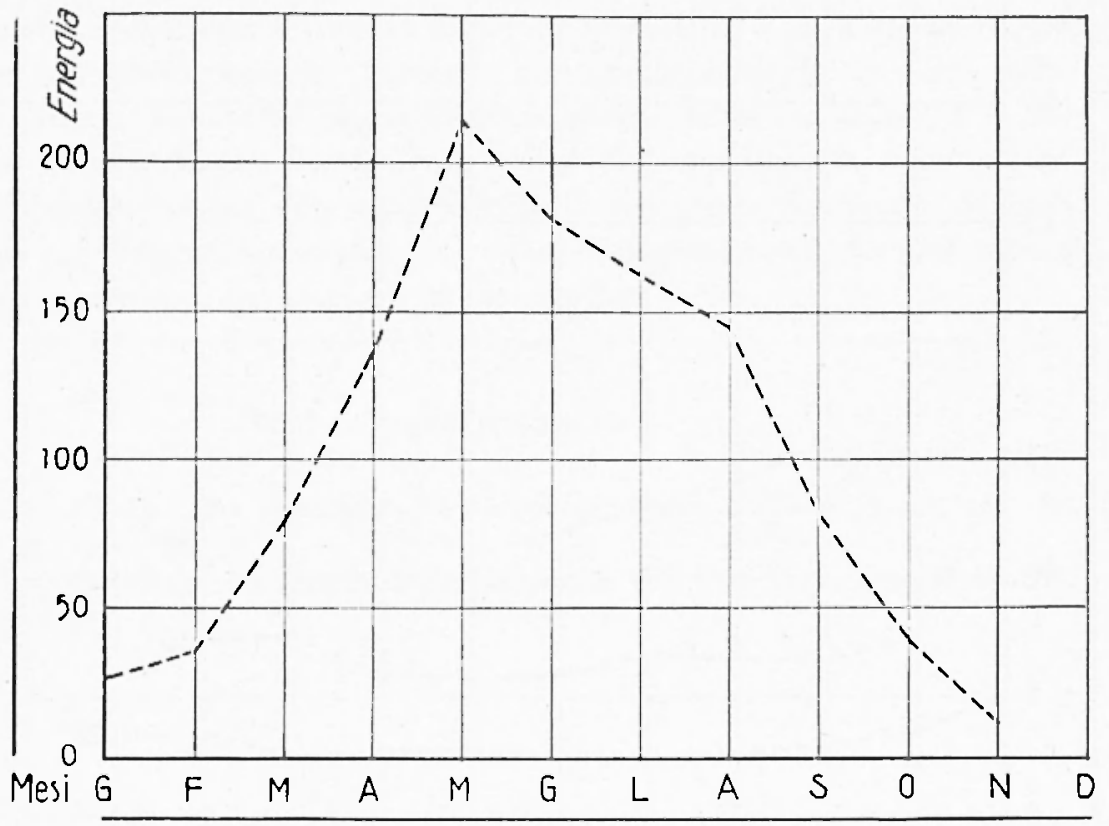

Fig. 1

go l'anno della intensità della radiazione $U V$ a Pavia per altezze di sole $20^{\circ}, 30^{\circ}, 40^{\circ}, 50^{\circ}$ e $60^{\circ}$. Come si vede la intensità della radiazione misurata con il dosimetro, per altezze superiori ai $40^{\circ}$, ha un minimo marcato in corrispondenza al mese di luglio (1945). Per i valori elevati dell'angolo di altezza, per i quali evidentemente la radiazione diffusa è meno importante in confronto di quella diretta, quel minimo di intensità non ha certamente relazione con il massimo del contenuto di ozono degli alti strati atmosferici; infatti se si guardano gli andamenti annui del contenuto di ozono misurati dal 1939 al $1943\left(^{1}\right)$ nelle stazioni di Arosa, Potsdam, Aahrus e Tromsö, una simile cor- 
rispondenza non si trova poiché il massimo nel contenuto di ozono si verifica secondo quelle misure, come pure secondo quelle di Dobson, nei mesi primaverili. A spiegare il disaccordo in questione probabilmente valgono ancora le considerazioni esposte prima. E tuttavia da segnalare il fatto che $\mathrm{i}$ risultati di recenti misure di radiazione solare $U V$ diretta, fatta a Locarno Monti, con cellula fotoelettrica al $\mathbf{C d}$ $(\lambda=0,288-0,366 \mu)$, fra l'ottobre 1945 e la fine del 1947, danno pure andamenti delle intensità $U V$ con l'altezza del sole che poco

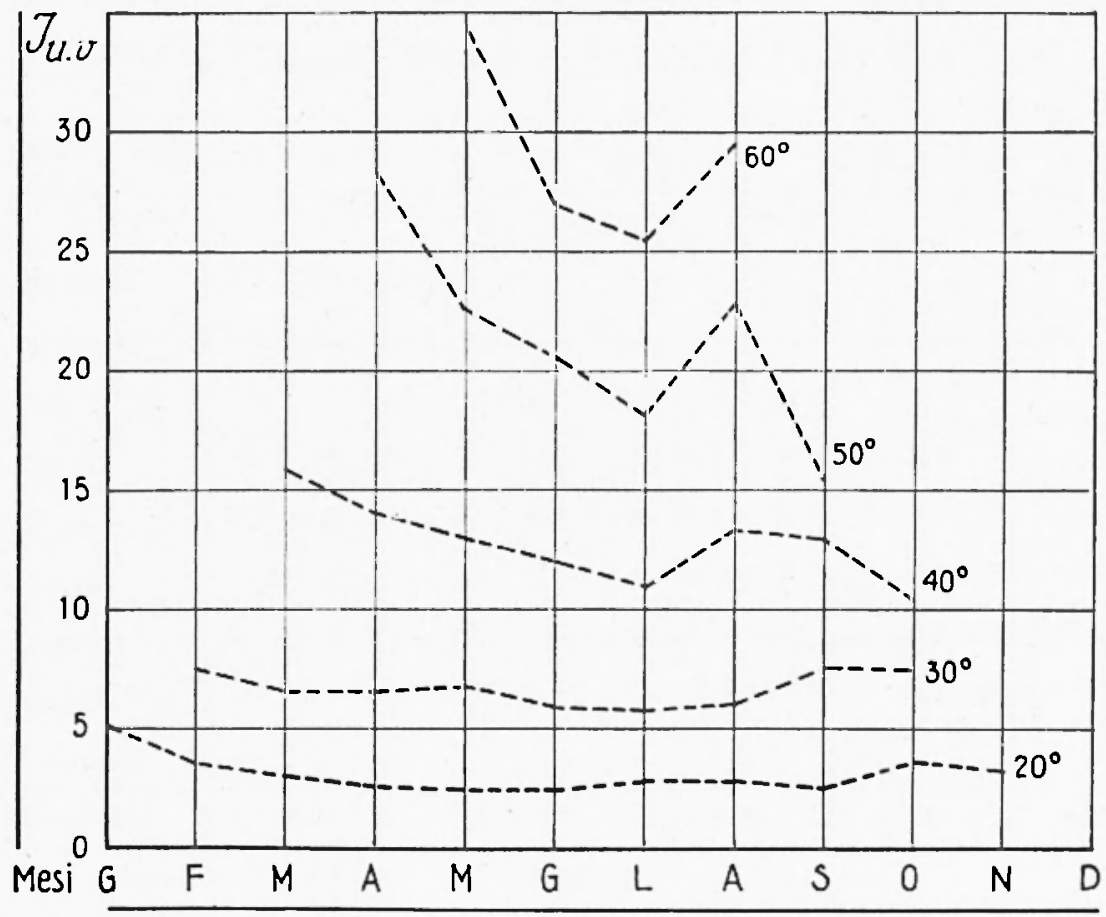

Fig. 2

hanno a che fare con gli andamenti del contenuto di ozono citati prima. D'altra parte anche altre misure di intensità dell' $C V$ solare al suolo eseguite con la cellula a $C d$ (campionata a Davos) ed elaborate da $F$. Lindholın $\left({ }^{2}\right)$ indicano per ciascuna altezza del sole fino a $50^{\circ}$ (misure svizzere) un minimo della radiazione durante l'estate, come appunto risulta dalle nostre misure con il dosimetro. Le misure calcolate da Lindholm sono relative ai periodi 1916-18 e 1921-28. Nellia sensibilità, dei diversi strumenti in questione, per lunghezze d'onda sensibilmente maggiori di $0,30 \mu$ si dovrà verosimilmente trovare la 
spiegazione dell'andamento annuo della intensità $U V$ misurata sia dal dosimetro sia dalla cellula a $C d$.

Napoli - Istituto Universitario Navale.

Istituto Naz. di Geofisica - Osserv. di Pavia - Febbraio 1951.

\section{RIASSUNTO}

Si pubblicano $i$ risultati di un anno di misure eseguite a Pavia, a cielo sereno, con il dosimetro per l'ultravioletto e si mette in rilievo come le nostre misure, d'accordo con quelle analoghe di altri sperimentatori, denuncino un andamento annuo della radiazione UV che non si accorda con l'andamento annuo del contenuto in ozono osservato dalle stazioni che seguono regolarmente tale contenuto. Si mettono pure in evidenza alcune condizioni sperimentali che potrebbero intervenire nel determinare il disaccordo.

\section{BIBLIOGRAFIA}

(1) Berichte des Deutschen Wetterdienstes in der US-Zone. N. 11, pag. 35, fig. $29,1949$.

(2) Gutesinerg V.: Handbuch der Geophysik. Vol. VIII, fig. 2, pag. 313, tab. 45, ediz. 1943. 